\title{
Liste des travaux cités en abregé
}

Agapitos, John Tzetzes : P. AgAPITOS, John Tzetzes and the Blemish Examiners: a Byzantine Teacher on Schedography, Everyday Language and Writerly Disposition. MEG 17 (2017) 1- 57.

AHRWEILER, Mer : H. AHRWEILER, Byzance et la mer. La marine de guerre, la politique et les institutions maritimes de Byzance aux VII $-\mathrm{XV}$ e siècles. Bibliothèque byzantine. Études, 5. Paris, 1966.

AlEXAKIS, The Modesty Topos : A. AlEXAKIS, The Modesty Topos and John of Damascus as a Not-SoModest Author. BZ 97 (2004) 521-530.

ALEXIOU, Literary subversion : M. ALEXIOU, Literary Subversion and the Aristocracy in Twelfth-Century Byzantium: A Stylistic Analysis of the Timarion (ch. 6-10). BMGS 8 (1982) 29-45.

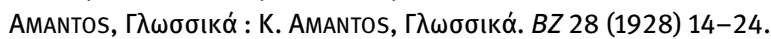

ANDERSOn / JefFReYs, The Decoration : J.C. AndeRSOn / M.J. JefFREYS, The Decoration of the Sevastokratorissa's Tent. Byz 64 (1994) 8-18.

ANGold, Church : M. ANGold, Church and Society in Byzantium under the Comneni, 1081-1261. Cambridge 1995.

Antiochos, Discours de consolation : A. SIDERAS, Die unedierte Trostrede des Gregorios Antiochos an den logothetes Michael Hagiotheodorites. JÖB 55 (2005) 147-190.

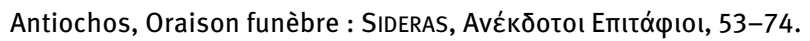

Aphthonios, Progymnasmata : PATILLON, Corpus Rhetoricum. I 47-162.

ARAGIONE, La transmission du savoir: G. ARAGIONE, La transmission du savoir entre «tradition » et «plagiat » dans l'Antiquité classique et chrétienne. Études de lettres 1-2 (2010) 117-138.

BALDWIN, Aspects of Byzantine Satire : B. BALDWIN, A Talent to Abuse: Some Aspects of Byzantine Satire. BF 8 (1982) 19-28.

BARDY, Eusèbe de Césarée, Histoire ecclésiastique : Eusèbe de Césarée Histoire ecclésiastique, éd. G. BARDY. III. SC, 55. Paris 1976.

Basilakès, Aristènos : GARZYA, Nicephorus Basilaces, 10-25.

Basilakès, Prologue : GARZYA, Nicephorus Basilaces, 1-9.

BeEs, Peloponnes und Hellas: N. BeES, Zur Sigillographie der byzantinischen Themen Peloponnes und Hellas. VV 21 (1914) 90-110, 192-235.

BeRnARD, The Ethics of Authorship : F. BeRnARD, The Ethics of Authorship: Some Tensions in the 11th Century, dans : PIZzonE, The Author, 41-60.

Bernardinello, Sicilia : S. Bernardinello, Sicilia e Normanni in Teodoro Prodromo, dans : ByzantinoSicula II. Miscellanea in memoria di G. Rossi Taibbi. Palermo 1975, 51-72.

BIAnconI, Qualcosa di nuovo su Giovanni Catrario: D. BIAnConI, Qualcosa di nuovo su Giovanni Catrario. MEG 6 (2006) 69-91.

BIRKENMEIER, Komnenian Army : J.W. BIRKENMEIER, The Development of the Komnenian Army 1081-1180. History of Warfare, 5. Leiden/Boston/Köln 2002.

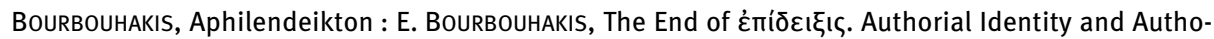

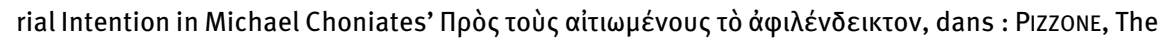
Author, 201-224.

BOURBOUHAKIS, The Epitaphios for Manuel I Komnenos by Eustathios of Thessalonike: E. BourBOUHAKIS, Not composed in a chance matter. The Epitaphios for Manuel I Komnenos by Eustathios of Thessalonike. Acta Universitatis Upsaliensis. Studia Byzantina Upsaliensia, 18. Uppsala 2017.

Bourbouhakis, Rhetoric and Performance : E. Bourbouhakis, Rhetoric and Performance, dans : STEPHENSON, The Byzantine World, 175-187. 
Browning, Patriarchal School: R. Browning, The Patriarchal School at Constantinople in the $12^{\text {th }}$ Century. Byz 32 (1962) 167-202; 33 (1963) 11-40 = idem, Studies on Byzantine History, Literature and Education. X. Variorum Collected Studies, 59. London 1977.

Bucossi / SuAREZ, John II Komnenos : A. BucossI / A. R. SUAREZ (éds.), John II Komnenos, emperor of Byzantium: in the shadow of father and son. Publications of the Centre of Hellenic Studies, 17. Farnham 2016.

BudeLMANN, John Tzetzes on ancient Greek literature : F. BUdeLMANN, Classical commentary in Byzantium: John Tzetzes on ancient Greek literature, dans : R.K. GIBSON / C. SHUTtLEWORTH KRAUS (éds.), The Classical Commentary: Histories, Practices, Theory. Mnemosyne Supplement, 232. Leiden /Boston/Köln 2002, 141-169.

CAmeron, Arguing: A. CAMERon, Arguing it out: Discussion in Twelfth-Century Byzantium. Budapest/New York 2016.

Campagnolo-Pothitou / Cheynet, Sceaux de la collection Georges Zacos: M. CAmpagnolo-Pothitou / J.- $\mathrm{Cl}$. CheYnet, Sceaux de la collection Georges Zacos au Musée d'art et d'histoire de Genève. Genève 2016.

CAPOnE CIOLlaro, L'Esegesi anonima alla Teogonia di Esiodo : M. CAPONE CIOLLARO, L'Esegesi anonima alla Teogonia di Esiodo in due codici napoletani. Atti dell'Accademia Pontaniana 30 (1981) 113128.

CAPONE CIOllaro, Forme e funzioni dell'Esegesi anonima alla Teogonia di Esiodo : M. CAPONE CIOllaro, Forme e funzioni dell'Esegesi anonima alla Teogonia di Esiodo, dans : F. ConCA (éd.), Byzantina Mediolanensia. V Congresso Nazionale di Studi Bizantini, Milano, 9-12 ottobre 1994. Atti. Milano 1996, 79-86.

CASPAR, Roger II : E. CASPAR, Roger II (1101-1154) und die Gründung der normannisch-sicilischen Monarchie. Innsbruck 1904.

CesArett, Allegoristi di Omero a Bisanzio : P. Cesaretti, Allegoristi di Omero a Bisanzio. Ricerche ermeneutiche (XI-XII secolo). Milano 1991.

CESARETTI / RONCHEY, Eustathii Exegesis : Eustathii Thessalonicensis exegesis in canonem iambicum pentecostalem, éds. P. CeSARETtI / S. RonCheY. Supplementa Byzantina. Texte und Untersuchungen, 10. Berlin/München/Boston 2014.

ChALANDON, Domination normande II : F. CHALANDON, Histoire de la domination normande en Italie et en Sicile. II. Paris 1907.

Chalandon, Les Comnène : F. ChalAndon, Les Comnène. Étude sur l'empire byzantin au XI et au XII siècles. II. Jean II Comnène (1118-1143) et Manuel I Comnène (1143-1180). Paris 1912.

Charitôn, De Callirhoe narrationes amatoriae : Chariton Aphrodisiensis, De Callirhoe narrationes amatoriae, éd. B.P. REARDON. Bibliotheca scriptorum Graecorum et Romanorum Teubneriana. München/Leipzig 2004.

CheYnet / Vannier, Études prosopographiques: J.- Cl. Cheynet / J.-Fr. VAnNier, Études prosopographiques. Byzantina Sorbonensia, 5. Paris 1986.

CheYnet, Philadelphie : J.-Cl. Cheynet, Philadelphie, un quart de siècle de dissidence, 1182-1206, dans : Philadelphie et autres études. Byzantina Sorbonensia, 4. Paris 1984, 39-54. $=i d e m$, The Byzantine Aristocracy and its Military Function. IX. Variorum Collected Studies Series, 859. Aldershot 2006.

CHEYNET / GÖKYILDIRIM / BuLgurLu, Sceaux d'Istanbul: J.-Cl. CHEYNET / T. GÖKYILDIRIM / V. BuRGURLU, LeS sceaux byzantins du Musée archéologique d'Istanbul. Istanbul 2012.

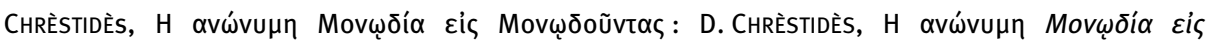

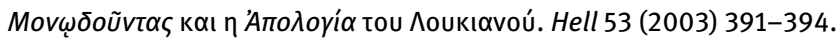

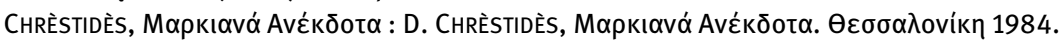




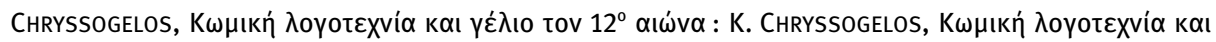

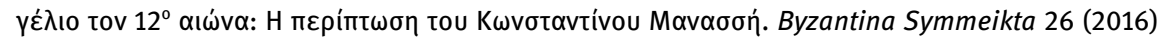
$141-161$.

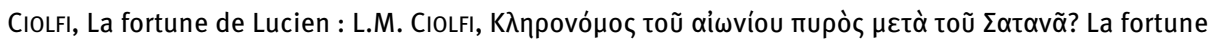
de Lucien entre sources littéraires et tradition manuscrite. Porphyra 24 (2015) 39-54.

Colonna, Himerii declamationes : A. ColonNA, Himerii declamationes et orationes cum deperditarum fragmentis. Scriptores Graeci et Latini. Roma 1951.

Companion to Archaic Greece : K.A. RAAFLAUB / H. van Wees (éds.), A Companion to Archaic Greece. Blackwell Companions to the Ancient World. Oxford 2009.

CONLEY, Byzantine criticism : T.M. CONLEY, Byzantine criticism and the uses of literature, dans : A. MINNIS / I. JOHNSON (éds.), The Cambridge History of Literary Criticism, II: The Middle Ages. Cambridge 2005, 669-692.

Constantinou, Female Corporeal Performances : S. Constantinou, Female Corporeal Performances: Reading the Body in Byzantine Passions and Lives of Holy Women. Acta Universitatis Upsaliensis. Studia Byzantina Upsaliensia, 9. Uppsala 2005.

Coufalova, The Noble Illustrious Warrior : H. Coufalova, The Noble Illustrious Warrior: Youths in Komnenian Literature. Graeco-Latina Brunensia 20 (2015) 3-17.

CozzA-LuZI : J. CozzA-LuzI, Sancti Theodori Studitae sermones magnae catecheseos. Nova Patrum Bibliotheca, 9/2. Roma 1888.

CULLHED, The blind Bard : E. CULLHED, The blind bard and 'l': Homeric biography and authorial personas in the twelfth century. BMGS 38 (2014) 49-67.

CULLhed, Parekbolai : Eustathios of Thessalonike Commentary on Homer's Odyssey, éd. E. CULLhED. I: On Rhapsodies A-B. Acta Universitatis Upsaliensis. Studia Byzantina Upsaliensia, 17. Uppsala 2016.

Cuomo, Ioannis Canani de Constantinopolitana obsidione relatio : A.M. Cuomo, Ioannis Canani de Constantinopolitana obsidione relatio. A Critical Edition, with English Translation, Introduction, and Notes of John Kananos' Account of the Siege of Constantinople in 1422. Byzantinisches Archiv, 30. Boston/Berlin 2016.

DARrouzès, L'éloge de Nicolas III : J. Darrouzżs, L'éloge de Nicolas III par Nicolas Mouzalon. RÉB 46 (1988) 5-53.

DARrouż̇s, Notice : J. Darrouzès, Notice sur Grégoire Antiochos (1160-1196). RÉB 20 (1962) 61$92=i d e m$, Littérature et histoire des textes byzantins. VII. Variorum Collected Studies Series, 10. London 1972.

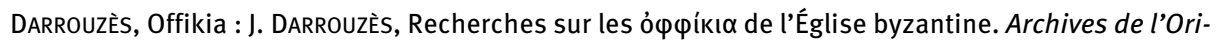
ent chrétien, 11. Paris 1970.

Darrouzès, Tornikès : J DARRouzÈs, Georges et Dèmètrios Tornikès, Lettres et Discours. Paris 1970.

DE ANDRÉs, Catálogo de los códices griegos de la Real Biblioteca de el Escorial : G. DE ANDRÉs, Catálogo de los códices griegos de la Real Biblioteca de el Escorial. II. Codices 174-420. Madrid 1965.

DE GREGoRIO, La spada di Alessio Contostefano : G. DE GREGoRIo, Teodoro Prodromo e la spada di Alessio Contostefano (Carm. Hist. LII Hörandner). Nea Rhome 7 (2010) 191-295.

Delehaye, Synaxarium ecclesiae Constantinopolitanae: H. DelehAYE, Synaxarium ecclesiae Constantinopolitanae e codice Sirmondiano nunc Berolinensi adiectis synaxariis selectis: Propylaeum ad Acta Sanctorum Novembris. Bruxelles 1902.

Delierneux, The Literary Portrait of Byzantine Female Saints: N. Delierneux, The Literary Portrait of Byzantine Female Saints, dans : ST. EFTHYMIADIS (éd.), The Ashgate Rechearch Companion to Byzantine Hagiography. II: Genres and Contexts. Farnham 2014, 363-386.

DöLGER / WIRTH / MÜLLER, Regesten : F. DÖLGER / P. WIRTH / A. MüLLER, Regesten der Kaiserurkunden des oströmischen Reiches: von 556-1453. II: Regesten von 1025-1204: mit Nachträgen zu Regesten 
Faszikel 3. Corpus der griechischen Urkunden des Mittelalters und der neueren Zeit, 1,2. München 1995.

Downey / Norman / SCHenkL, Themistii orationes: G. Downey / A.F. Norman / H. SCHenKL, Themistii orationes quae supersunt. I-III. Bibliotheca scriptorum Graecorum et Romanorum Teubneriana. Leipzig 1965-1974.

EIDENEIER, Ptochoprodromos: H. EIDENEIER, Ptochoprodromos. Einführung, kritische Ausgabe, deutsche Übersetzung, Glossar. Köln 1991.

Eusebios, Praep. evan. : SCHROEDER / Des PLACES, Eusèbe de Césarée, La préparation évangelique.

Eusebios, Hist. eccl. : BARDY, Eusèbe de Césarée, Histoire ecclésiastique.

Eustathe de Thessalonique, Comm. ad Hom. Il. : VAN DER VALK.

Eustathe de Thessalonique, Comm. ad Hom. Odys. : Stallbaum

Eustathe de Thessalonique, ep. : F. Kolovou, Die Biefe des Eustathios von Thessalonike. Einleitung, Regesten, Text, Indizes. Beiträge zur Altertumskunde, 239. Berlin 2006.

Eustathe de Thessalonique, Michael Hagiothéodôritès : WIRTH, Eustathii Thessalonicensis Opera Minora, 141-151.

Eustathe de Thessalonique, Oraison funèbre de Nikolaos Hagiothéodôritès : WIRTH, Eustathii Thessalonicensis Opera Minora, 3-16.

Eustathe de Thessalonique, Or. 2 : WIRTH, Eustathii Thessalonicensis Opera Minora, 17-45.

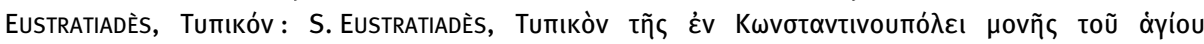

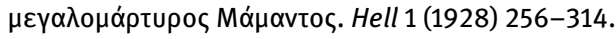

ÉVIEUX : Isidore de Péluse, Lettres. II. Lettres 1414-1700, éd. P. ÉVIEUX. SC, 454. Paris 2000.

FLACH, Glossen : H. FLACH, Glossen und Scholien zur hesiodischen Theogonie mit Prolegomena. Leipzig 1876.

FosKolou, Decoding Byzantine ekphraseis : V. FosKOLOU, Decoding Byzantine ekphraseis on works of Art. Constantine Manasses's description of earth and its audience. BZ 111 (2018) 71-102.

Gagliano, Hermes Propylaios: E. Gagliano, Hermes Propylaios (e le Charites) sull'Acropoli di Atene. Annuario della scuola archeologica di Atene e delle missioni italiane in Oriente 92 ser. III, 14 (2014) 33-67.

GAISFORD, Poetae minores Graeci : T GAISFORD, Poetae minores Graeci. II. Scholia ad Hesiodum. Leipzig 1823.

Gallay, Lettres : P. GallaY, Saint-Grégoire de Nazianze, Lettres. I. Collection des Universités de France Serie grecque - Collection Budé, 165. Paris 1964.

GARZYA, Literariche und rhetorische Polemiken : A. GARZYA, Literariche und rhetorische Polemiken der Komnenenzeit. BSI 34 (1973) 1-14= idem, Storia e interpretazione di testi bizantini. Saggi e ricerche. VII. Variorum Collected Studies Series, 28. London 1974.

GARZYA, Nicephorus Basilaces : Nicephori Basilacae Orationes et Epistolae, éd A. GARZYA. Bibliotheca scriptorum Graecorum et Romanorum Teubneriana. Leipzig 1984.

GARZYA, Synésios de Cyrène Lettres : Synésios de Cyrène. II et III. Correspondance : Lettres I-CLVI, éd. A. GARZYA, trad. D. ROQUES. Collection des Universités de France Série grecque - Collection Budé, 397. Paris 2000.

GaUL, Thomas Magistros : N. GaUL, Thomas Magistros und die spätbyzantinische Sophistik: Studien zum Humanismus urbaner Eliten in der frühen Palaiologenzeit. Mainzer Veröffentlichungen zur Byzantinistik, 10. Wiesbaden 2011.

GaUL, Perfomative Reading in the Late Byzantine Theatron : N. GAUL, Perfomative Reading in the Late Byzantine Theatron, dans : SHAWCROSS / TоTH, Reading in the Byzantine Empire, 215-233.

GAUtIER, L'édit : P. GAUTIER, L'édit d'Alexis Ier sur la réforme du clergé. RÉB 31 (1973) 165-201.

GAUTIER, Michel Italikos : P. GAUTIER, Michel Italikos, Lettres et Discours. Archives de l'Orient chrétien, 14. Paris 1972. 
GAUTIER, Théophylacte d'Achrida Discours : P. Gautier, Théophylacte d'Achrida, Discours, Traités, Poésies : Introduction, Texte, Traduction et notes. CFHB, 16/1. Thessalonique 1980.

GAUTIER, Théophylacte d'Achrida Lettres : P. GAUTIER, Théophylacte d'Achrida, Lettres : Introduction, Texte, Traduction et notes. CFHB, 16/2. Thessalonique 1986.

GAUTIER, Typikon Pantokratôr : P. GAUTIER, Le Typikon du Christ Sauveur Pantocrator. RÉB 32 (1974) 1145.

Gentile Messina, Epitafio : R. Gentile Messina, Basilio Achrideno. Epitafio per l'imperatrice alamanna. Introduzione, testo critico, traduzione e commento. Pubblicazioni del Centro studi sull'antico cristianesimo, 3. Catania ${ }^{2} 2008$.

Giannoul, Balianites: A. Giannoul, Leon Balianites, Exegetische Didaskalien. Zur Interpunktion im Codex Escorialensis Y II 10, dans : A. GIANnoul / E. SCHIFFER (éds.), From Manuscripts to Book / Vom Codex zur Edition. Proceedings of the International Workshop on Textual Criticism and Editorial Practice for Byzantine Texts (Vienna, 10-11 December 2009). Denkschriften. Österreichische Akademie der Wissenschaften, phil.-hist. Klasse, 431. Veröffentlichungen zur Byzanzforschung, 29. Wien 2011, 79-84.

GOAR, Euchologion : J. GOAR, EủXo ${ }^{\prime}$ ýyov sive rituale Graecorum complectens ritus et ordines divinae liturgiae, officiorum, sacramentorum, consecrationum, benedictionum, funerum, orationum, etc. cuilibet personae, statui, vel tempori congruos, juxta usum orientalis ecclesiae, cum selectis Bibliothecae Regiae, Barberinae, Cryptae-Ferratae, Sancti Marci Florentini, Tillianae, Allatianae, Coresianae et aliis probatis MM. SS. et editis exemplaribus collatum. Venetiae ${ }^{2} 1730$.

GouILLARD, Synodikon : J. GouILLARD, Le Synodikon de l'orthodoxie : Édition et commentaire. TM 2 (1967) 1-316.

Greg. Naz., Or. 8 : Grégoire de Nazianze, Discours 6-12, éd. M.-A. CALVEt-SeBASTI. SC, 405. Paris 1995, 246-299.

Grég. Naz., Or. 27 : Grégoire de Nazianze, Discours 27-31, éd. P. GallaY avec la collaboration de M. JOURJON. SC, 250. Paris 1978, 70-99.

Grég. Naz., Or. 36 : Grégoire de Nazianze, Discours 32-37, éd. C. MoReSCHINI / P. GaLLAY. SC, 318. Paris 1985, 240-269.

Grég. Naz., Or. 43 : Grégoire de Nazianze, Discours 42-43, éd. J. BeRNARDI. SC, 384. Paris 1992, 116307.

Grégoire de Nysse, Contra Eunomium III : Gregorii Nysseni Contra Eunomium Libri. Pars altera: Liber III (vulgo III-XII). Refutatio Confessionis Eunomii (vulgo lib. II), éd. W. JAEGER. Gregorii Nysseni Opera, 2. Leiden 1960.

Grègoras, Historia: Nicephori Gregorae Byzantina Historia, éd. I. BEKKER / L. SCHOPEN. I-III. CSHB. Bonn 1829-1855.

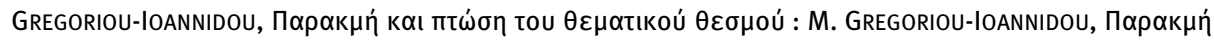

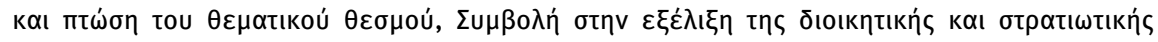

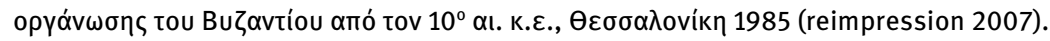

GRIMAL, Dictionnaire : P. GRIMAL, Dictionnaire de la mythologie grecque et romaine. Paris 1951.

GRÜNBART, Reconstructing networks in 12th century Byzantium: M. GRÜNBART, 'Tis love that has warm'd us. Reconstructing networks in 12th century Byzantium. Revue belge de philologie et d'histoire 83 (2005) 301-313.

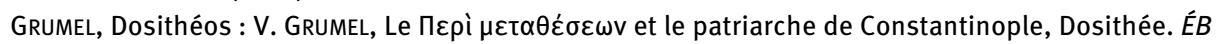
1 (1943) 239-249.

GRUMEL / DARROUZĖs, Regestes : V. Grumel / J. DARROuZĖs, Les regestes des actes du patriarcat de Constantinople. I. Les actes des patriarches. II-III. Les regestes de 716-1206. Paris 1989.

GUILLAND, Le grand domestique: R. GuILLAND, Le grand domestique, dans: GuILLAND, Recherches, I 405-425. 
GUILLAND, Le protovestiaire : R. GUILLAND, Fonctions et dignités des eunuques. II. Le protovestiaire. ÉB 2 (1944) 202-220 = idem, Recherches, I 216-236.

GUILLAND, Les chefs de la marine : R. GUILLAND, Études de titulature et de prosopographie byzantines. Les chefs de la marine byzantine : Drongaire de la flotte, Grand Drongaire de la flotte, Duc de la flotte, Mégaduc. BZ 44 (1951) 212-240 = idem, Recherches I 535-562.

GUILLAND, Les logothètes : R. GUILLAND, Les logothètes. Études sur l'histoire administrative de l'Empire byzantin. RÉB 29 (1971) 5-115.

GUILLAND, Recherches : R. GUILLAND, Recherches sur les institutions byzantines. I-II. Berliner byzantinistische Arbeiten, 35. Berlin/Amsterdam 1967.

HALDON, Warfare : J. HALDON, Warfare, State and Society in the Byzantine World, 560-1204. Warfare and History. London 1999.

HALSALL, Life of Saint Thomaïs of Lesbos : P. HALSALL, Life of Saint Thomaïs of Lesbos, dans : TALBot, Holy Women of Byzantium, 291-322.

Heath, Pseudo-Dionysius Art of Rhetoric: M. Heath, Pseudo-Dionysius Art of Rhetoric 8-11. Figured Speech, Declamation and Criticism. American Journal of Philology 124 (2003) 81-107.

Hérmogène, De id. : PATILLON, Corpus Rhetoricum IV.

HeRrIN, Realities : J. HeRrIN, Realities of Byzantine Provincial Government: Hellas and Peloponnesos, 1180-1205. DOP 29 (1975) 253-284 = eadem, Margins and Metropolis: Authority across the Byzantine Empire. Princeton/Oxford 2013, chap. 3, 58-102 (mise à jour de la bibliographie).

HinTERBERgER, Autobiographische Traditionen: M. HINTERBERGER, Autobiographische Traditionen in Byzanz. WBS, 22. Wien 1999.

HÖRANDNER, Historische Gedichte : W. Hörandner, Theodoros Prodromos: Historische Gedichte. WBS, 11. Wien 1974.

HÖRANDNER, La poésie : W. HöRANDNER, Forme et fonction. Remarques sur la poésie dans la société byzantine. Séminaires byzantins, 14. Paris 2017.

HÖRANDNER, Pseudo-Gregorios Korinthios : W. HÖRANDNER, Pseudo-Gregorios Korinthios “Über die vier Teile der perfekten Rede". MEG 12 (2012) 87-132.

HÖRANDNER, Zur Beschreibung von Kunstwerken : W. HÖRANDNER, Zur Beschreibung von Kunstwerken in der byzantinischen Dichtung - am Beispiel des Gedichts auf das Pantokratorkloster in Konstantinopel, dans : C. RATKOWITSCH (éd.), Die poetische Ekphrasis von Kunstwerken. Eine literarische Tradition der Großdichtung in Antike, Mittelalter und früher Neuzeit. Österreichische Akademie der Wissenschaften, Sitzungsberichte der phil.-hist. Klasse, 735. Wien 2006, 203-219.

HÖRANDNER / GRÜNBART, L'épistolographie et la poésie épigrammatique : W. HÖRANDNER / M. GRÜNBART (éds.), L'épistolographie et la poésie épigrammatique : projets actuels et questions de méthodologie. Actes de la $16^{\mathrm{e}}$ Table Ronde organisée par W. Hörandner et M. Grünbart dans le cadre du XX Congrès international des Études byzantines, Collège de France-Sorbonne, Paris 19-25 août 2001. Dossiers Byzantins, 3. Paris 2003.

HOHLWEG, Beiträge : A. HOHLWEG, Beiträge zur Verwaltungsgeschichte des Oströmischen Reiches unter den Komnenen. Miscellanea Byzantina Monacensia, 1. München 1965.

HORNA, Konstantinos Manasses : K. HORNA, Eine unedierte Rede des Konstantin Manasses. WSt 28 (1906) 171-204.

Houben, Roger II of Sicily : H. Houben, Roger II of Sicily: A Ruler Between East and West. Cambridge 2002.

HUNGER, Allegorische Mythendeutung : H. HUnGER, Allegorische Mythendeutung in der Antike und bei Johannes Tzetzes. JÖBG 3 (1954) $35-54=$ idem, Byzantinische Grundlagenforschung. Gesammelte Aufsätze. XIV. Variorum Collected Studies Series, 21. London 1973.

HUnger, Athen in Byzanz : H. HUnGER, Athen in Byzanz: Traum und Realität. JÖB 40 (1990) 43-61.

HUNGER, Johannes Tzetzes, Allegorien : H. HUNGER, Johannes Tzetzes, Allegorien aus der Verschronik. Kommentierte Textausgabe. JÖBG 4 (1955) 13-49. 
HUNGER, Hochsprachliche Literatur : H. HUNGER, Die hochsprachliche profane Literatur der Byzantiner. I. Philosophie, Rhetorik, Epitolographie, Geschichtsschreibung, Geographie. Handbuch der Altertumswissenschaft. Abteilung XII. Byzantinisches Handbuch, 5. München 1978.

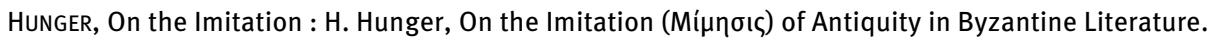
DOP 23/24 (1969/1970) 15-38 = idem, Byzantinische Grundlagenforschung. Gesammelte Aufsätze. XV. Variorum Collected Studies Series, 21. London 1973.

Ioannes Mauropous, ep. : KARPOZILOS.

Isidôros Pèlousiôtès, ep. : Évieux.

JANIN, Églises et Monastères : R. JANIN, La géographie ecclésiastique de l'Empire byzantin. I. Le siège de Constantinople et le patriarcat oecuménique. III. Les églises et les monastères. Paris ${ }^{2} 1969$.

Jouanno, Women in Byzantine Novels of the $12^{\text {th }}$ century : C. Jouanno, Women in Byzantine Novels of the $12^{\text {th }}$ century : An Interplay between Norm and Fantasy, dans : L. GARLAND (éd.), Byzantine Women. Varieties of Experience, 800-1200. Aldershot 2006, 141-162.

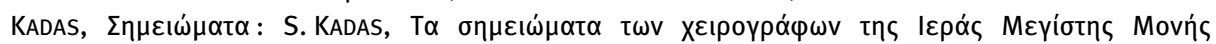
Ватопаıठ́íou. 'Ayıov 'Opos 2000.

KaldeluIS, The Christian Parthenon : A. Kaldellis, The Christian Parthenon. Classicism and Pilgrimage in Byzantine Athens. Cambridge/New York 2009.

KALDeluIS, Classical Scholarship : A. KALDELLIS, Classical Scholarship in Twelfth-Century Byzantium, dans : C. BARBER / D. JeNKINS (éds.), Medieval Greek Commentaries on the Nicomachean Ethics. Studien und Texte zur Geistesgeschichte des Mittelalters, 101. Leiden/Boston 2009, 1-43.

KALDELLIS, Hellenism : A. KALDELLIS, Hellenism in Byzantium. The Transformation of Greek Identity and the Reception of the Classical Tradition. Greek Culture in the Roman World. Cambridge/New York 2007.

Kalliklés : R. Romano, Nicola Callicle Carmi. Testo critico, introduzione, traduzione, commentario e lessico. Byzantina et Neo-hellenica Neapolitana Collana di Studi e Testi, 8. Napoli 1980.

KARLSSON, Idéologie: G. KARLSSON, Idéologie et cérémonial dans l'épistolographie byzantine. Textes $\mathrm{du} \mathrm{X}^{\mathrm{e}}$ siècle analysés et commentés : Nouvelle édition, revue et augmenté. Acta Universitatis Upsaliensis. Studia Graeca Upsaliensia, 3. Uppsala 1962.

KARPOZILOS : A. KARPOZILOS, The Letters of Ioannes Mauropous Metropolitan of Euchaita. Greek Text, Translation, and Commentary. CFHB, 34. Thessalonike 1990.

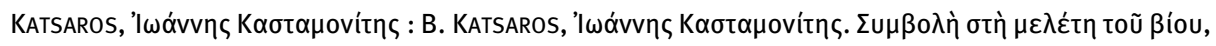

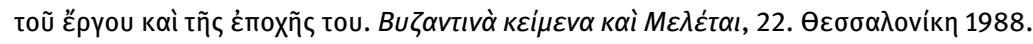

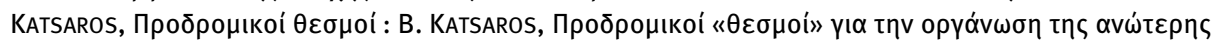

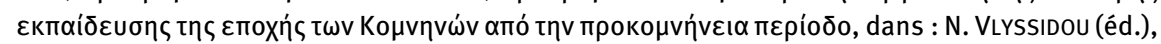
The Empire in Crisis? Byzantium in the $11^{\text {th }}$ Century (1025-1081). National Hellenic Research Foundation/Institute for Byzantine Research. International Symposium, 11. Athens 2003, 443471.

KAZHDAN, Brat'ja Ajofeodority : A.P. KAZHDAN, Brat'ja Ajofeodority pri dvore Manuila Komnina. ZRVI 9 (1966) 85-94.

KAZHDAN / FrankLin, Studies : A.P. KAZHDAN / S. FrankLIN, Studies on Byzantine Literature of the $11^{\text {th }}$ and $12^{\text {th }}$ Centuries. Past and Present Publications. Cambridge/Paris 1984.

Kazhdan / Wharton Epstein, Change : A.P. Kazhdan/A. Wharton Epstein, Change in Byzantine Culture in the 11th and 12th Centuries. Berkeley/Los Angeles/London 1985.

KAZHDAN / RONCHEY, L'aristocrazia bizantina : A.P. KAZHDAN / S. RONCHEY, L'aristocrazia bizantina dal principio dell'XI alla fine del XII secolo. Palermo 1997.

Kinnamos : Ioannis Cinnami Epitome rerum ab loanne et Alexio Comnenis Gestarum, éd. A. MEINEKE. CSHB. Bonn 1836.

KODER / HILD, Hellas und Thessalia : J. KOdeR / F. HILD, Hellas und Thessalia. TIB, 1. Wien 1976. 
Kolovou, Michael Choniates: Michaelis Choniatae Epistulae, éd. F. Kolovou. CFHB, 41. Berlin/New York 2001.

KotZABASSI, Feasts: S. KotZABASSI, Feasts at the Monastery of Pantokrator, dans: KotZABASSI Pantokrator, 153-189.

KotzabassI, Pantokrator : S. KotzABASSI (éd.), The Pantokrator Monastery in Constantinople. Byzantinisches Archiv, 27. Boston/Berlin 2013.

Krallis, Harmless satire : D. KRallis, Harmless Satire, Stringing Critique : Notes and Suggestions for Reading the Timarion, dans : D. ANGELOV / M. SAXBY, Power and Subversion in Byzantium. Papers from the 43rd Spring Symposium of Byzantine Studies, University of Birmingham, March 2010. Publication of the Society for the Promotion of Byzantine Studies, 17. Farnham 2013, 221-246.

KRESTEN, Theodoros Styppeiotes: O. KRESTEN, Zum Sturz des Theodoros Styppeiotes. JÖB 27 (1978) 49-103.

KRUMBACHER : K. KRUMBACHER, Geschichte der byzantinischen Literatur von Justinian bis zum Ende des oströmischen Reiches (527-1453). Handbuch der klassischen Altertums-Wissenschaft in systematischer Darstellung mit besonderer Rücksicht auf Geschichte und Methodik der einzelnen Disziplinen. München ${ }^{2} 1897$.

LAIOU, Life of Saint Mary the Younger : A. LAIOU, Life of Saint Mary the Younger, dans : TALBOT, Holy Women of Byzantium, 239-289.

LAIOU, Mariage : A.E. LAIOU, Mariage, amour et parenté à Byzance aux XIe-XIII siècles. Paris 1992.

LAIOU, The Role of Women in Byzantine Society : A.E. Laiou, The Role of Women in Byzantine Society. JÖB 31/1 (1981) 233-260 = eadem, Gender, Society and Economic Life in Byzantium. I (with addendum). Variorum Collected Studies Series, 370. Aldershot 1992 = C. BEATTIE (éd.), Women in the Medieval World. 4. Abington 2017, 206-230.

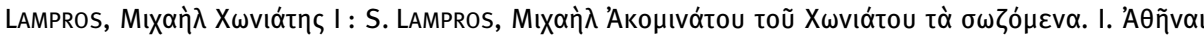
1879.

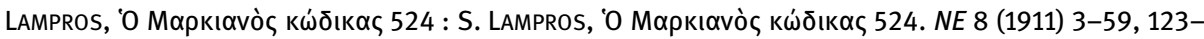
192.

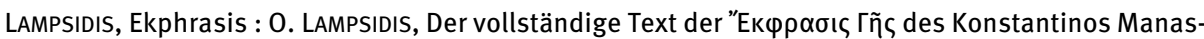
ses. JÖB 41 (1991) 189-205.

LARSON, Ancient Greek Cults : J. LARSON, Ancient Greek Cults: A Guide. New York/London 2007.

LARSON, Greek Nymphs : J. LARSON, Greek Nymphs: Myth, Cult, Lore, Oxford/New York 2001.

LAURENT, Deux nouveaux gouverneurs de la Bulgarie byzantine : V. LAURENT, Deux nouveaux gouverneurs de la Bulgarie byzantine : Le proèdre Nicéphore Batatzès et le protoproèdre Grégoire. $R E$ SEE 7, 1 (1969) 143-150.

LAURENT, Kataphloros : V. LAURENT, Kataphloros, patronyme supposé du métropolite de Thessalonique Eustathe. RÉB 20 (1962) 218-221.

LAURENt, Regestes : V. LAURENT, Les regestes des actes du patriarcat de Constantinople, I. Les actes des patriarches. IV. Les regestes de 1208 à 1309. Paris 1971.

LAURENT, Sceaux : V. LAURENT, Le corpus des sceaux de l'empire byzantin. II. L'administration centrale. Paris 1981.

LAURENT, Sceau du Basile Kamatèros : V. LAURENT, Un sceau inédit du protonotaire Basile Kamatéros, Byz 6 (1934) 253-272.

LEFORT, Prooimion : J. LEFORT, Prooimion de Michel, neveu de l'archevêque de Thessalonique, didascale de l'Évangile. TM 4 (1970) 375-393.

LEONE, Dramation : P.L.M. LEONE, Michaelis Hapluchiris versus cum excerptis. Byz 39 (1969) 251-283.

LILIE, Byzanz und die Kreuzfahrerstaaten : R.-J. LILIE, Byzanz und die Kreuzfahrerstaaten. Studien zur Politik des byzantinischen Reiches gegenüber den Staaten der Kreuzfahrer in Syrien und Palästina bis zum vierten Kreuzzug (1096-1204). Poikila Byzantina, 1. München 1981. 
LILIE, Handel : R.-J. LILIE, Handel und Politik zwischen dem byzantinischen Reich und den italienischen Kommunen Venedig, Pisa und Genua in der Epoche der Komnenen und der Angeloi (1081-1204). Amsterdam 1984.

LIVERANI, Sul sistema di interpunzione in Eustazio di Tessalonica : I.A. LIVERANI, Sul sistema di interpunzione in Eustazio di Tessalonica. MEG 1 (2001) 187-197.

LOUKAKI, Antiochos : M. LOUKAKI, Grégoire Antiochos. Éloge du patriarche Basile Kamatèros. Texte, traduction, commentaire suivis d'une analyse des œuvres de Grégoire Antiochos. Byzantina Sorbonensia, 13. Paris 1996.

LOUKAKI, Collaborators : M. LOUKAKI, Empress Piroska-Eirene's Collaborators in the Foundation of the Pantokrator Monastery: The Testimony of Nikolaos Kataphloron, dans : KoTZABASSI, Pantokrator, 191-201.

LOUKAKI, Douze didascales : M. LOUKAKI, Remarques sur le corps de douze didascales au XII" siècle, dans : EYYYXIA. Mélanges offerts à Hélène Ahrweiler. II. Byzantina Sorbonensia, 16. Paris 1998, 427-438.

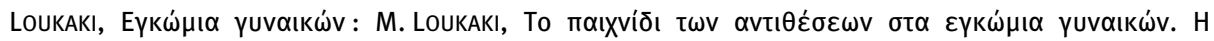

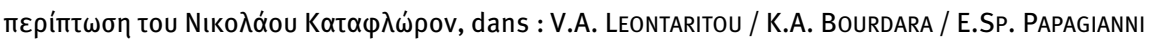
(éds.), Antecessor. Festschrift für Spyros N. Troianos zum 80. Geburtstag. Athen 2013, 895-915.

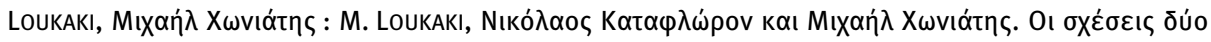

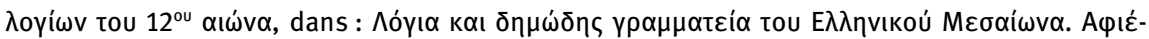

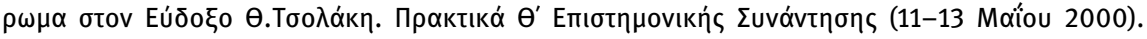

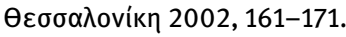

LOUKAKI, Patronyme : M. LOUKAKI, Kataphlôros ou Kataphlôron : Notes sur un patronyme byzantin. ZRVI 50 (2013) 357-365.

LOUKAKI, Le samedi de Lazare : M. LOUKAKI, Le samedi de Lazare et les éloges annuels du patriarche de

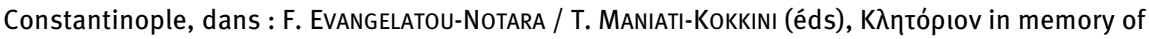
Nikos Oikonomides. Athens/Thessaloniki 2005, 327-346.

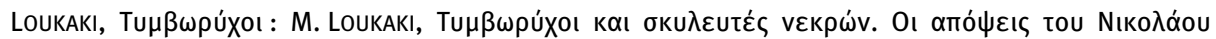

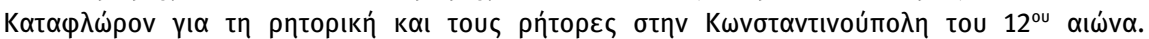
Symmeikta 14 (2001) 143-166.

LOUKAKI, Xiphilin : M. LOUKAKI, Discours annuels en l'honneur du patriarche Georges Xiphilin. Textes édités et commentés; traduits par C. JouAnNo. Collège de France-CNRS. Centre de recherche d'histoire et civilisation de Byzance. Monographies, 18. Paris 2005.

MACRIDES / MAGDALINO, The architecture of ekphrasis : R.J. MACRIDES/P. MAGDALINO, The Architecture of Ekphrasis: Construction and Context of Paul the Silentiary's Poem on Hagia Sophia. BMGS 12 (1988) 47-82.

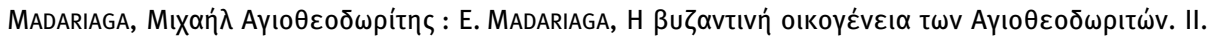

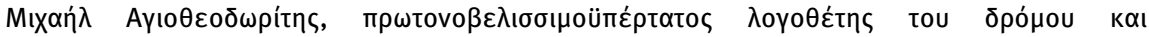
орфаvотрóфоৎ. Byzantina Symmeikta 24 (2014) 213-246.

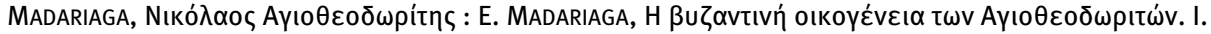

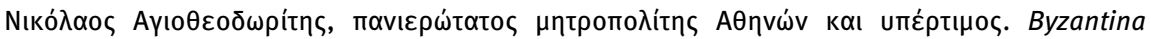
Symmeikta 19 (2009) 147-181.

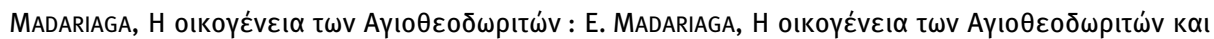

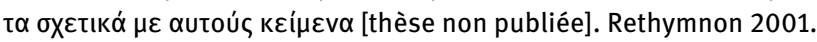

Magdalino, Bagoas : P. MAgdalino, The Bagoas of Nikephoros Basilakes: a Normal Reaction, dans : L. MAYALI / M.M. MART, Of Strangers and Foreigners (Late Antiquity - Middle Ages). Berkeley 1993, 47-63.

Magdalino, Dérision : P. Magdalino, Tourner en dérision à Byzance, dans : E. CRouzet-PAVAn / J. Verger (éds.), La dérision au Moyen Âge. Cultures et civilisations médiévales, 38. Paris 2007, 55-72. 
Magdalino, Hellenism and Nationalism : P. MAgdalino, Hellenism and Nationalism in Byzantium, dans : idem, Tradition and Transformation in Medieval Byzantium. XIV. Variorum Collected Studies Series, 343. Aldershot 1991, 1-27.

Magdalino, Manuel : P. Magdalino, The Empire of Manuel I Komnenos (1143-1180). Cambridge 1993.

MAgdalino, Pantokrator Monastery : P. MAGDALINO, The Foundation of the Pantokrator Monastery in its Urban Setting, dans : KOTZABASSI, Pantokrator, 33-55.

Magdalino, The Reform Edict : P. Magdalino, The Reform Edict of 1107, dans : M. Mullett / D. SMYthe (éds.), Alexios I Komnenos. Papers of the $2^{\text {nd }}$ Belfast Byz. Intern. Colloquium, 14-16 April 1989. BBTT 4.1. Belfast 1996, 199-218.

MAKK, Árpáds : F. MAKK, The Árpáds and the Comneni: Political Relations between Hungary and Byzantium in the 12th Century. Budapest 1989.

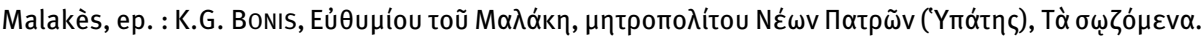

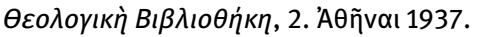

Malakès, Oraison funèbre d'Alexis Kontostéphanos : PAPADOPOULOS-KERAMEUS, Noctes, 142-154.

Malakès, Oraison funèbre de Nikolaos Hagiothéodôritès : PAPADOPOULOS-KERAMEUS, Noctes, $154-162$.

MALHERBE, Ancient Epistolography Theorists : A.J. MALHERBE, Ancient Epistolography Theorists. Society of Biblical Litterature. Sources for Biblical Study, 19. Atlanta 1988.

MANGo / PARKER, Description : C. MANGo / J. PARKER, A Twelfth Century Description of St. Sophia. DOP 14 (1960), 233-245.

Manuel Holobôlos, Or. 1 : Manuelis Holoboli orationes, éd. M. TREU. Programm des königlichen Victoria-Gymnasiums, 2. Potsdam 1907, 51-78.

MARCINIAK, Bion Prasis: P. MARCINIAK, Theodoros Prodromos' Bion Prasis: A Reappraisal. GRBS 53 (2013) 219-239.

MARCINIAK, The Byzantine Performative Turn : P. MARCINIAK The Byzantine Performative Turn, dans : K. TwARDowSKA et a. (éds.), Within the Circle of Ancient Ideas and Virtues. Studies in Honour of Professor Maria Dzielska. Krakow 2014, 423-430.

MARCINIAK, Byzantine Theatron : P. MARCINIAK, Byzantine Theatron - A Place of Performance?, dans : M. GRÜNBARt (éd.), Theatron. Rhetorische Kultur in Spätantike und Mittelalter. Millennium Studien/Millenium Studies, 13. Berlin/New York 2007, 277-285.

MARCINIAK, Reception of Ancient Literature : P. MARCINIAK, The Undead in Byzantium. Some Notes on the Reception of Ancient Literature in Twelfth-Century Byzantium. Troianalexandrina 13 (2013) 95-111.

MARCINIAK, Reinventing Lucian in Byzantium : P. MARCINIAK, Reinventing Lucian in Byzantium. DOP 70 (2016) 209-224.

MARCINIAK, Satire and Invective in Byzantine Literature : P. MARCINIAK, The Art of Abuse: Satire and Invective in Byzantine Literature. A preliminary Survey. Eos 103 (2016) 349-362.

MARCINIAK / WARCABA, Ekphrasis of a chariot race : P. MARCINIAK / K. WARCABA, Racing with Rhetoric: a Byzantine ekphrasis of a chariot race. BZ 107 (2014) 97-112.

MazzUCCHI, Ambrosianus : C.M. MAZzUCCHI, Ambrosianus C 222 inf. (Graecus 886): Il codice e il suo autore. Aevum 78 (2004) 441-440.

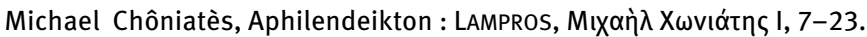

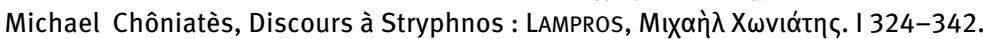

Michael Chôniatès, ép. : Kolovou.

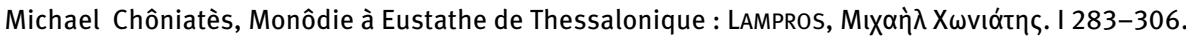

Michael de Thessalonique : REgEL, Fontes, 131-182.

Michael Psellos, Chronographie : Michaelis Pselli Chronographia, éd. D.R. REINSCH. I. Einleitung und Text. Millenium-Studien/Millenium Studies, 51. Berlin/Boston 2014. 
MIGLIORINI, Gli scritti satirici di Teodoro Prodromo : T. MIGLIORINI, Gli scritti satirici in greco letterario de Teodoro Prodromo: Introduzione, edizione, traduzione e commenti. Pisa 2010 (thèse non publiée).

MILLER, Catalogue des manuscrits grecs de la bibliothèque de l'Escurial : E. Miller, Catalogue des manuscrits grecs de la bibliothèque de l'Escurial. Paris 1848.

MÜLLER : K. MüLLER, Geographi Graci minores. II. Paris 1861.

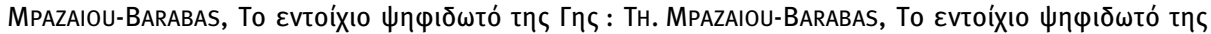

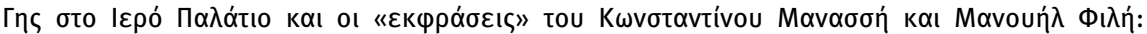

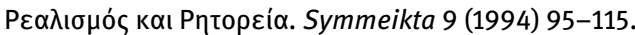

MuLlett, Aristocracy : M. Mullett, Aristocracy and Patronage in the Literary Circles of Comnenian Constantinople, dans : M. ANGOLD (éd.), The Byzantine Aristocracy, IX to XIII Centuries. Oxford 1984, 173-201 = eadem, Letters, Literacy and Literature in Byzantium. VIII. Variorum Collected Studies Series, 889. Aldershot 2007.

MULLETT, The Detection of Relationship in Middle Byzantine Literary Texts : M. MuLLETT, The Detection of Relationship in Middle Byzantine Literary Texts: The Case of Letters and Letter-networks, dans : HÖRANDNER / GRÜNBART, L'épistolographie et la poésie épigrammatique, 63-74.

Mullett, Epistolography: M. Mullett, Epistolography, dans : E. JefFreYs / J. HALDon / R. CORMACK (éds.), The Oxford Handbook of Byzantine Studies. Oxford 2008, 884-893.

Mulletr, Friendship in Byzantium : M. Mullem, Friendship in Byzantium: Genre, Topos and Network, dans : J. HASELDINE (éd.), Friendship in Medieval Europe. Stroud 1999, 166-184.

MuLletr, Object, Text and Performance : M. MulletT, Object, Text and Performance in Four Komnenian Tent Poems, dans : SHAWCROSS / TOTH, Reading in the Byzantine Empire, 414-423.

NESBITT / WIITA, Confraternity : J. NESBITT / J.J. WIITA, A Confraternity of the Comnenian Era. BZ 68 (1975) 360-384.

Niederau, Veneto-byzantinische Analekten : K. NiederaU, Veneto-byzantinische Analekten zum byzantinisch-normannischen Krieg 1147-1158. Aachen 1982.

Nikétas Chôniatès, Historia : Nicetae Choniatae, Historia, éd. I.A. van DIETEN. CFHB, 11/1. Berlin/New York 1975.

Nikétas Chôniatès, Oraison funèbre de Théodoros Trochos : Nicetae Choniatae Orationes et Epistulae, éd. I.A. van DieTEN. CFHB, 3. Berlin/New York 1972, 13-25.

NILSSON / ZAGKLAS, The Use of Greek Novels : I. NILSSON / N. ZAGKLAS, "Hurry up, reap every flower of the logoi". The Use of Greek Novels in Byzantium. GRBS 57 (2017) 1120-1148.

Nikolaos le sophiste, Progymnasmata : Nicolai Progymnasmata, éd. J. FELTEN. Bibliotheca scriptorum Graecorum et Romanorum Teubneriara, Rhetores Graeci, 11. Leipzig 1913, 1-79.

NÜNLIST, Homer as Blueprint : R. NÜNLIST, Homer as a Blueprint for Speechwriters: Eustathius' Commentaries and Rhetoric. GRBS 52 (2012) 493-509.

OIKONOMIDÈs, Fiscalité : N. OIKONOMIDÈs, Fiscalité et exemption fiscale à Byzance (IXe-XI ${ }^{\mathrm{e}} \mathrm{s}$.). Fondation Nationale de la Recherche Scientifique. Institut de Recherches Byzantines. Monographies, 2. Athènes 1996.

OIKONOMIDÈs, The Role of the Byzantine State in the Economy : N. OIKONOMIDĖs, The Role of the Byzantine State in the Economy, dans : A. LAlOu (éd.), The Economic History of Byzantium: From the Seventh through the Fifteenth Century, III. DOS, 39. Washington, D.C. 2002, 973-1058.

OUSTERHOUT, Architecture : R. OUSTERHOUt, Architecture and patronage in the age of John II, dans: BucossI / SUAREZ, John II Komnenos, 135-154.

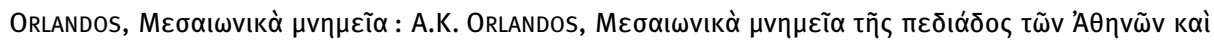

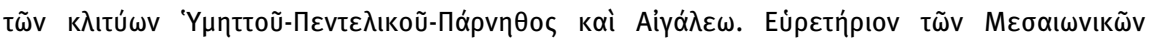

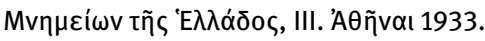

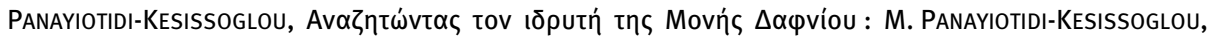

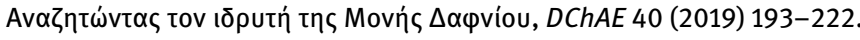




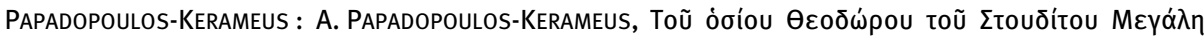

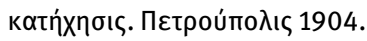

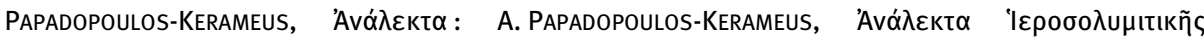

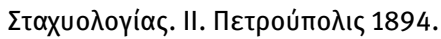

Papadopoulos-Kerameus, Noctes: A. Papadopoulos-Kerameus, Noctes Petropolitanae. Sbornik vizantijskich tekstov XII-XIII věkov. Sankt Petersburg 1913.

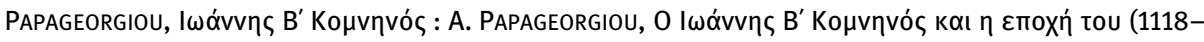
1143). Aөńva 2017.

Papaloannou, Letter-Writing : S. Papaloannou, Letter-Writing, dans : StePHenson, The Byzantine World, 188-199.

Papaloannou, Psellos : S. Papaioannou, Michael Psellos: Rhetoric and Authorship in Byzantium. Cambridge 2013.

Papaloannou, Voice, Signature, Mask : S. Papaloannou, Voice, Signature, Mask: The Byzantine Author, dans : PIZZONE, The Author, 21-40.

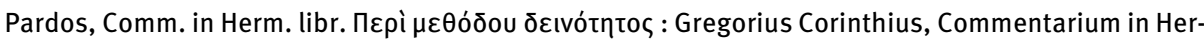

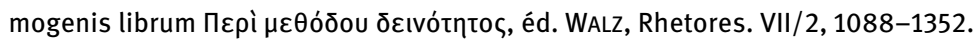

Patillon, Corpus Rhetoricum I: M. Patillon, Corpus Rhetoricum. I. Anonyme, Préambule à la rhétorique. Aphthonios, Progymnasmata. En annexe: Pseudo-Hermogène, Progymnasmata. Collection des Universités de France Série grecque - Collection Budé, 460. Paris 2008.

PatILlon, Corpus Rhetoricum III : M. PATILLon, Corpus Rhetoricum. III, $1^{\text {re }}$ partie. Pseudo-Hermogène, L'Invention - Anonyme, Synopse des Exordes. Collection des Universités de France Série grecque - Collection Budé, 485. Paris 2012

Patillon, Corpus Rhetoricum IV : M. Patillon, Corpus Rhetoricum. IV. Prolégomènes au De Ideis Hérmogène, Les catégories stylistiques du discours (De Ideis) - Synopse des éxposés sur les Ideai, Collection des Universités de France Série grecque - Collection Budé, 491. Paris 2012.

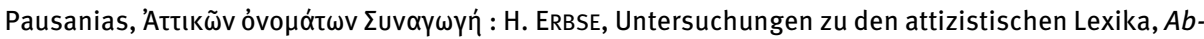
handlungen der deutschen Akademie der Wissenschaften zu Berlin, phil.-hist. Kl. 1949.2. Berlin 1950, 152-221.

Pernot, Periautologia : L. Pernot, Periautologia. Problèmes et méthodes de l'éloge de soi-même dans la tradition éthique et rhétorique grécoromaine. RÉG 111 (1998) 101-124.

PERNOT, La Rhétorique dans l'Antiquité : L. PERNOT, La Rhétorique dans l'Antiquité. Paris 2000.

PETIT, Le monastère de Notre-Dame de Pitié : L. PEtIT, Le monastère de Notre-Dame de Pitié en Macédoine. IRAIK 6 (1900) 1-153.

Photios, Bibliothèque : Photius, Bibliothèque. III : Codices 186-222, éd. R. HenRY. Collection des Universités de France Série grecque - Collection Budé, 158. Paris 1962.

Pizzone, The Author: A. PIzzone (éd.), The Author in Middle Byzantine Literature: Modes, Functions and Identities. Byzantinisches Archiv, 28. Boston/Berlin 2014.

PIzzone, Eustathios' Commentaries : A. PIzzone, Lady Phantasia's "Epic" Scrolls and Fictional Creativity in Eustathios' Commentaries on Homer. MEG 14 (2014) 177-198.

PIzzone, The Prolog of Nikephoros Basilakes : A. PIzzone, Anonymity, Dispossession and Reappropriation in the Prolog of Nikephoros Basilakes, dans : PIZZONE, The Author, 225-243.

PODESTÀ, Le satire lucianesche di Teodoro Prodromo : G. PODESTÀ, Le satire lucianesche di Teodoro Prodromo. Aevum 19 (1945) 239-252; 21 (1947) 3-25.

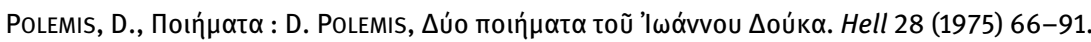

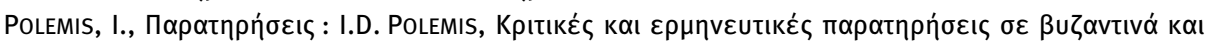

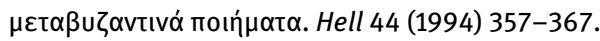

Polemis, Constantine Psaltopoulos : I.D. PolemIS, Constantine Psaltopoulos and his Unpublished Address to Michael Hagiotheodorites. BF 21 (1995) 159-165. 


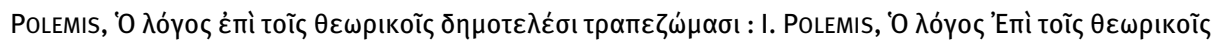

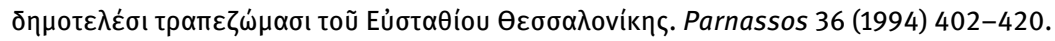

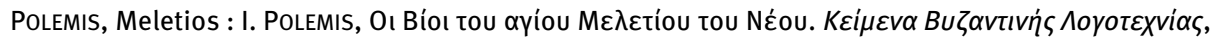
9. A日ńva 2018.

Porphyrius, Vita Pythagorae : E. DES PlACES (avec la contribution de A.-PH. SECONDS), Vie de Pythagore - Lettre à Marcella. Collection des Universités de France Série grecque-Collection Budé, 285. Paris 1982.

PRATSCH, Der hagiographische Topos : T. PRATSCH, Der hagiographische Topos: griechische Heiligenviten in mittelbyzantinischer Zeit. Millenium-Studien/Millenium Studies, 6. Berlin/New York 2005.

Prieto Domínguez, Saint Theokleto : O. Prieto Domínguez, Saint Theokleto: A Female Iconoclast Saint. AnBoll 134 (2016) 293-302.

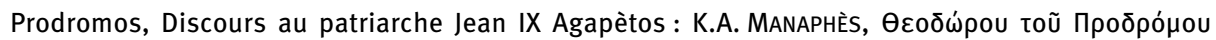

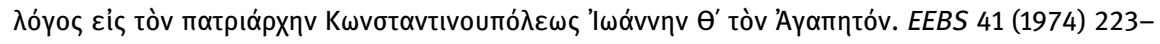
242.

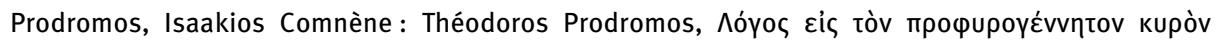

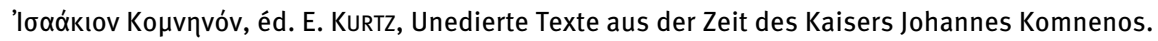
BZ 16 (1907) 112-119.

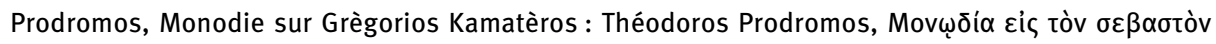

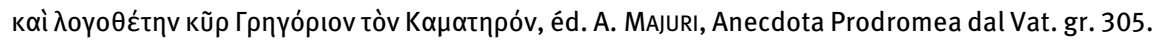
Rendiconti della Reale Accademia dei Lincei. Classe di scienze morali, storiche e filologiche. Ser. V 17. Roma 1908, 528-535.

Prodromos, Rhodanthè et Dosiclés : Theodori Prodromi De Rhodanthes et Dosiclis amoribus libri IX, éd. M. MARCOVICH. Bibliotheca scriptorum Graecorum et Romanorum Teubneriana. Stuttgart 1992.

Prodromos, Vie de saint Mélétios : Polemis, Meletios.

Pryor / JefrReYs, The Byzantine Navy : J. Pryor / E. JefrReYs, The Age of the $\Delta \rho{ }^{\prime} \mu \omega v$. The Byzantine Navy ca 500-1204. The Medieval Mediterranean Peoples, Economies and Cultures 400-500, 62. Leiden/Boston 2006.

Pseudo-Hermogène, De inv. : PATILLoN, Corpus Rhetoricum. III/1. VII-132.

Pseudo-Hermogène, Progymnasmata : PATILLon, Corpus Rhetoricum. I 164-206.

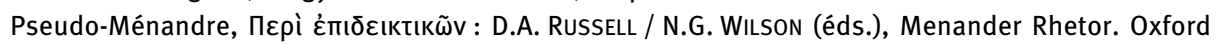
1981, 76-223 (texte), 271-361 (commentaire).

RASsow, Zum byzantinisch-normannischen Krieg: P. RASsow, Zum byzantinisch-normannischen Krieg 1147-1149. Mitteilungen des Instituts für Österreichische Geschichtsforschung 62 (1954) 213-218.

Regel, Fontes : W. RegeL, Fontes rerum Byzantinarum. Fasciculi 1 et 2. Rhetorum saeculi XII orationes politicae. Petropoli 1917 (réimpr. Leipzig 1982).

REINSCH, What Should an Editor Do with a Text Like the 'Chronographia' of Michael Psellos? : D. REIN$\mathrm{SCH}$, What Should an Editor Do with a Text Like the Chronographia of Michael Psellos?, dans : A. BucosSI / E. KIHLMAN, Ars Edendi Lectures Series. II. Acta Universitatis Stockholmiensis. Studia latina Stockholmiensia, 58. Stockholm 2012, 131-154.

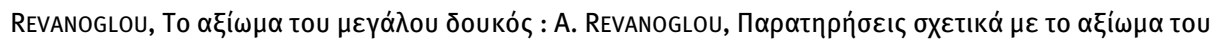

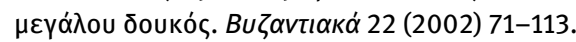

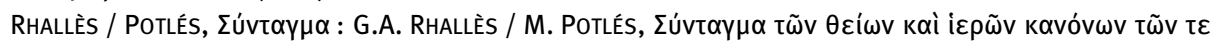

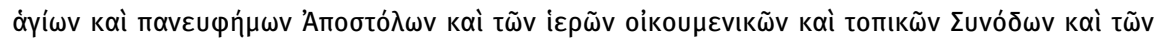

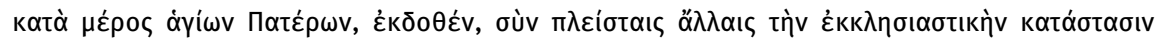

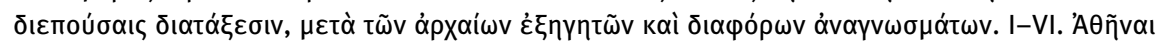
1852-1859. 
RHовY, War Athen Ziel des Normannenangriffes : A. RHOBY, War Athen Ziel des Normannenangriffes von 1147/1148? JÖB 52 (2002) 221-230.

Rнову, Reminiszenzen an antike Stätten : A. Rнову, Reminiszenzen an antike Stätten in der mittelund spätbyzantinischen Literatur. Eine Untersuchung zur Antikenrezeption in Byzanz. Göttinger Studien zur byzantinischen und neugriechischen Philologie, 1. Göttingen 2003.

RHOBY, Synesios von Kyrene als literarisches Vorbild : A. RHOBY, Synesios von Kyrene als literarisches Vorbild: Ep. 136 (Garzya) und der Ausgangspunkt der Athenklage, dans : HÖRANDNER / GRÜNBART, L'épistolographie et la poésie épigrammatique, 85-96.

RHOBY / SCHIFFER (éds.), Imitatio-Aemulatio-Variatio : A. RHOBY / E. SCHIFFER (éds.), Imitatio-AemulatioVariatio: Akten des Internationalen Wissenschaftlichen Symposions zur byzantinischen Sprache und Literatur (Wien, 22-25 Oktober 2008). Denkschriften. Österreichische Akademie der Wissenschaften, phil.-hist. Klasse, 402. Wien 2010.

Rollos, Amphoteroglossia : P. Rollos, Amphoteroglossia. A Poetics of the Twelfth-Century Medieval Greek Novel. Hellenic Studies, 10. Washington, D.C. 2005.

RoILos, Ancient Epics in Byzantium : P. RolLos, "Unshapely Bodies and Beautifying Embellishments": The Ancient Epics in Byzantium, Allegorical Hermeneutics, and the Case of loannes Diakonos Galenos. JÖB 64 (2014) 231-246.

RoILoS, Reconstructing the Implied Audience : P. RoILos, "I grasp, oh artist, your enigma, I grasp your drama": Reconstructing the Implied Audience of the $12^{\text {th }}$ Century Byzantine Novel, dans: C. CUPANE / B. KRÖNUNG (éds.), Fictional Storytelling in the Medieval Eastern Mediterranean and Beyond. Brill's Companions to the Byzantine World, 1. Leiden/Boston 2016, 463-478.

Romano, La satira bizantina: R. Romano, La satira bizantina dei secoli XI-XV: Il patriota, Caridemo, Timarione, Cristoforo di Mitilene, Michele Psello, Teodoro Prodromo, Carmi ptocoprodromici, Michele Haplucheir, Giovanni Catrara, Mazaris, La messa del glabro, Sinassario del venerabile asino. Classici greci. Torino 1999.

Romano, Timarion : R. Romano, Pseudo-Luciano, Timarione: testo critico, introduzione, traduzione, commentario e lessico. Byzantina et Neo-Hellenica Neapolitana Collana di Studi e Testi, 2. Napoli 1974.

Sarantènos, De philosophia et rhetorica: Manuel Sarantènos, De philosophia et rhetorica, éd. U. CRISCUOLo, Un opuscolo inedito di Manuele Karanteno o Saranteno. EEBS 42 (1975-1976) 213221.

SCHNEIDER, Concision et art épistolaire dans la lettre 51 de Grégoire de Nazianze : J. SCHNEIDER, Concision et art épistolaire dans la lettre 51 de Grégoire de Nazianze. Eruditio Antiqua 6 (2014) 1-12.

SCHÖNAUER, Quadragesima: S. SCHÖNAUER, Eustathios von Thessalonike, Reden auf die große Quadragesima: Prolegomena, Text, Übersetzung, Kommentar, Indices. Meletemata, 10. Frankfurt a. M. 2016.

SCHRoeder / Des Places, Eusèbe de Césarée, La préparation évangelique: G. SCHRoeder / E. Des PLACES, Eusèbe de Césarée La préparation évangelique, Livres VIII-IX-X. SC, 369. Paris 1991.

SEIBT, Bleisiegel : W. SEIBT, Die byzantinischen Bleisiegel in Österreich. I. Kaiserhof. Österreichische Akademie der Wissenschaften. Veröffentlichungen der Kommission für Byzantinistik, 2. Wien 1978.

SHAWCROSS / TOTH, Reading in the Byzantine Empire : T. SHAWCROSS / I. Тотн (éds.), Reading in the Byzantine Empire and Beyond. Cambridge 2018.

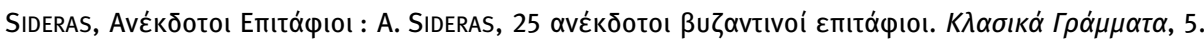

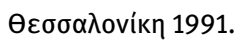

SIDERAS, Grabreden : A. SIDERAS, Die byzantinischen Grabreden. Prosopographie, Datierung, Überlieferung; 142 Epitaphien und Monodien aus dem byzantinischen Jahrtausend. WBS, 19. Wien 1994. 
SIDERAS, Invektive : A. SIDERAS, Eine byzantinische Invektive gegen die Verfasser von Grabreden.

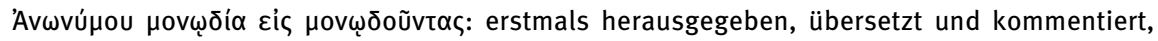
nebst einem Anhang über den rhythmischen Satzschluß. WBS, 23. Wien 2002.

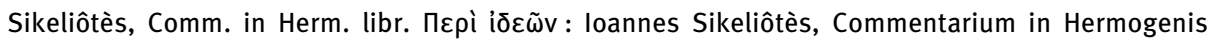

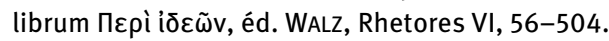

SIMPSON, Niketas Choniates : A. SIMPSON, Niketas Choniates : A Historiographical Study. Oxford Studies in Byzantium. Oxford 2013.

SPINGOU, Words and artworks : F. SPINGOU, Words and artworks in the twelfth century and beyond. The thirteenth-century manuscript Marcianus gr. 524 and the twelfth-century dedicatory epigrams on works of art. PhD Thesis. University of Oxford 2012 (thèse non publiée).

Stallbaum : Eustathii archiepiscopi Thessalonicensis Commentarii ad Homeri Odysseam, éd. J.G. Stallbaum. II. Leipzig 1826.

StANTON, Norman naval operations: C.D. StANTON, Norman Naval Operations in the Mediterranean. Warfare in history. Woodbridge 2011.

StaVRaKos, Familiennamen : C. STAVRAKOS, Die byzantinischen Bleisiegel mit Familiennamen aus der Sammlung des Numismatischen Museums Athen. Mainzer Veröffentlichungen zur Byzantinistik, 4. Wiesbaden 2000.

Stemplinger, Das Plagiat : E. Stemplinger, Das Plagiat in der griechischen Literatur. Berlin 1912.

StePhenson, The Byzantine World : P. StePHenson (éd.), The Byzantine World. London/New York 2010. SteRnBACH, Spicilegium : L. STERnBACH, Spicilegium Prodromeum. Cracovia 1904 (= Rozprawy Aka-

demii Umiejętności. Wydziat filologiczny serya II, 24 (1904) 336-368).

Suda : Suidae Lexicon, éd. A. AdLER. I-V. Lexicographi Graeci. Leipzig 1928-1938.

SYKOUTRIS, Epistolographie : I. SYKOUTRIS, art. Epistolographie, dans : RE Suppl. 5. [1931] 185-220.

Synésios de Cyrène, ep. : GARZYA, Synésios de Cyrène Lettres.

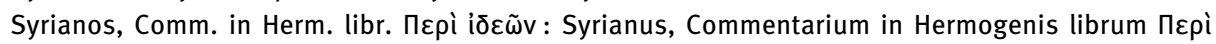

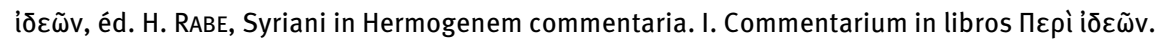
Bibliotheca scriptorum Graecorum et Romanorum Teubneriana. Leipzig 1892.

TAfEL, Opuscula : G.L.F. TAfEL, Eustathius Thessalonicensis, Opuscula. Accedunt Trapezuntinae historiae scriptores Panaretus et Eugenicus. Frankfurt a. M. 1832.

TAFT, Women at Church in Byzantium : R.F. TAFT, Women at Church in Byzantium. DOP 52 (1998) 2787.

TALBOT, Female Sanctity : A.-M. TALBOT, Sanctity and Hagiography: Female Sanctity in Byzantium, dans : eadem, Women and Religious Life in Byzantium. VI. Variorum Collected Studies Series, 733. Aldershot 2001, 1-16 [traduction en anglais de l'article Essere donna e santa, dans: S. GENTILE, Oriente cristiano e santità. Figure e storie di santi tra Bisanzio e l'Occidente. Milano 1998, 61-68].

TALBOT, Holy Women of Byzantium : A.-M. TALBOT (éd.), Holy Women of Byzantium. Ten Saints Lives in English Translation. Washington, D.C. 1996.

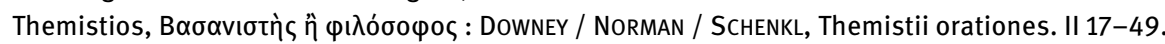

Themistios, $\Delta \varepsilon \kappa \varepsilon$ tпрıкós: DoWNEY / NORMAN / SCHENKL, Themistii orationes. I 215-230.

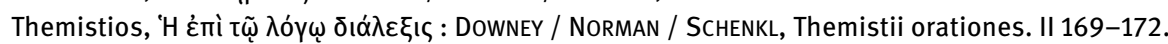

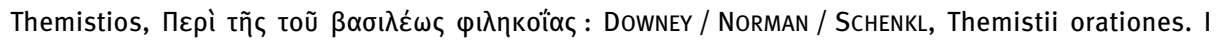
311-325.

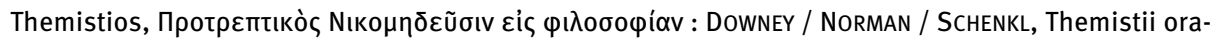
tiones. II 97-111.

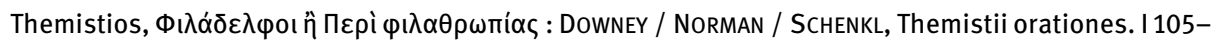
205.

Théodoros Stouditès, Catéchèses : PAPAdopoulos-Kerameus, CozzA-LuzI. 
Théôn, Progymnasmata: Aélius Théon, Progymnasmata, éd. M. PAtilton avec la contribution de G. BOLOGNESI. Collection des Universités de France Série grecque - Collection Budé, 376. Paris 1977.

Théophylaktos d'Achrida, ep. : GAUTIER, Théophylacte d'Achrida Lettres.

TREU, Schulgespräch : M. TREU, Ein byzantinisches Schulgespräch. BZ 2 (1893) 96-105.

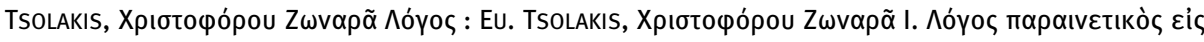

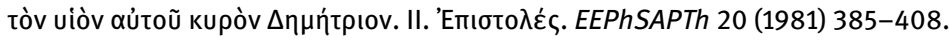

Tzétzès, Chiliades : Ioannis Tzetzae Historiae, éd. P.A.M. LEONE. Pubblicazioni dell'Istituto di filologia classica, 1. Napoli 1968.

Tzétzès, Comm. in Nub.: Scholia in Aristophanem, Pars 4. Johannes Tzetzae Commentarii Aristophanem. Fasc. II, Commentarium in Nubes, éd. D. HolwERDA. Scripta Academica Groningana. Groningen 1960.

Tzétzès, Ep. : Ioannis Tzetzae Epistulae, éd. P.A.M. LEONE. Bibliotheca scriptorum Graecorum et Romanorum Teubneriana. Leipzig 1972.

VAN DEN BERG, Homer and the Good Ruler: B. VAN DEN BERG, Homer and the Good Ruler in 'Age of Rhetoric': Eustathios of Thessalonike on Excellent Oratory, dans : J. KLOOSTER / B. VAN DEN BERG, Homer and the Good Ruler in Antiquity and Beyond. Leiden/Boston 2018, 219-238.

VAN DER VALK : Eustathii Archiepiscopi Thessalonicensis Commentarii ad Homeri lliadem Pertinentes, éd. M. van DER VALK. I-IV. Leiden 1971-1987.

VASSIS, Das Pantokratorkloster : I. VASSIS, Das Pantokratorkloster von Konstantinopel in der byzantinischen Dichtung, dans : KotZABASSI, Pantokrator, 203-249.

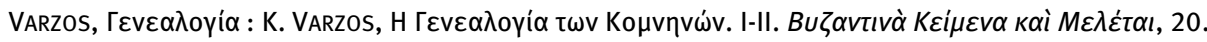

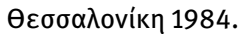

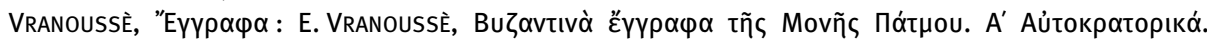

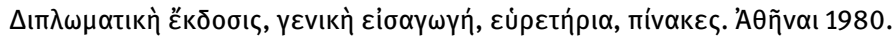

WALZ, Rhetores : C. WALZ, Rhetores Graeci. I-IX. Stuttgart/Tübingen 1832-1836.

WASSILIOU / SEIBT, Bleisiegel in Österreich : A.-K. WASSILIOU / W. SEIBT, Die byzantinischen Bleisiegel in Österreich. II. Zentral- und Provinzialverwaltung. Österreichische Akademie der Wissenschaften, Veröffentlichungen der Kommission für Byzantinistik 2/2. Denkschriften der philos.-histor. Klasse, 324. Wien 2004.

WASSILIOU-SEIBT, Corpus, I-II : A.-K. WASSILIOU-SEIBT, Corpus der byzantinischen Siegel mit metrischen Legenden. I. Einleitung. Siegellegenden von Alpha bis inklusive My. II. Siegellegenden von Ny bis inklusive Sphragis. WBS, 28/1-2. Wien 2011-2016.

WASSILIOU-SEIBT, Der Name Alexios Kontostephanos Komnenos : A.-K. WASSILIOU-SEIBT, Der Name Alexios Kontostephanos Komnenos. Identifizierungsvorschläge anhand sigillographischer Funde. Studies in Byzantine Sigillography, 10. Berlin/New York 2010, 25-32.

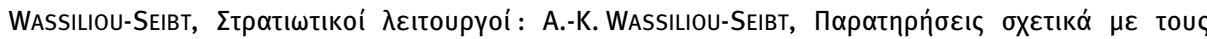

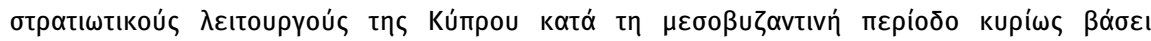

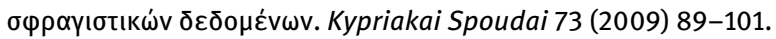

WEBB, Aesthetics of sacred space : R. WEBB, The Aesthetics of Sacred Space: Narrative, Metaphore, and Motion in "Ekphraseis" of Church Buildings. DOP 53 (1999) 59-75.

WEBB, Ekphrasis : R. WEBB, Ekphrasis, Imagination and Persuasion in Ancient Rhetorical Theory and Practice. Farnham 2009.

WEBB, Progymnasmata : R. WEBB, The progymnasmata as practice, dans : YUN LEE Too (éd.), Education in Greek and Roman Antiquity. Leiden/Boston/Köln 2001, 289-316.

WILSON, Scholarly Hands of the Middle Byzantine Period: N. WILSON, Scholarly Hands of the Middle Byzantine Period, dans : J. GLÉNISSON / J. BOMPAIRE / J. IRIGOIN (éds.), La paléographie grecque et byzantine: Paris 21-25 octobre 1974. Actes du Colloque international sur la paléographie 
grecque et byzantine. Colloques internationaux du Centre National de la Recherche Scientifique, 559. Paris 1977, 221-239.

WILSON, Three Byzantine Scribes : N. WILSON, Three Byzantine Scribes. GRBS 14 (1973) 223-228.

WIRTH, Die sprachliche Situation : P. WIRTH, Die sprachliche Situation in dem umrissenen Zeitalter. Renaissance des Attizismus. Herausbildung der neugriechischen Volkssprache, dans : XV Congrès International d’Études Byzantines. Rapports et co-rapports. II. Langue, Littérature, Philologie. Athènes 1976, 1-54.

WIRTH, Eustathiana: P. WIRTH, Eustathiana. Gesammelte Aufsätze zu Leben und Werk des Metropoliten Eustathios von Thessalonike. Amsterdam 1980.

WIRTH, Eustathii Thessalonicensis Opera Minora : P. WIRTH, Eustathii Thessalonicensis Opera Minora. CFHB, 32. Berlin/New York 2000.

WIRTH, Michael von Thessalonike? : P. WIRTH, Michael von Thessalonike?. BZ 55 (1962) 266-268.

WIRTH, Michael “von Konstantinopel” : P. WIRTH, Michael "von Konstantinopel” und kein Ende. Byz 37 (1967) 421-422.

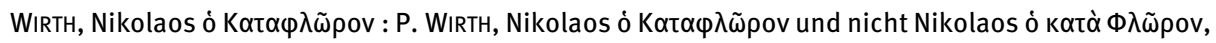

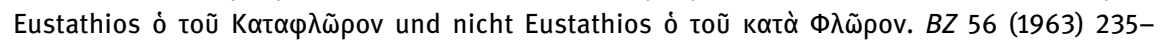
$236=$ idem, Eustathiana 5-6.

WIRTH, Zu Nikolaos Kataphloros : P. WIRTH, Zu Nikolaos Kataphloros. Classica et Mediaevalia 21 (1960) 212-214.

ZIEGLER, Plagiat : K. ZIEGLER, art. Plagiat, dans : RE 20, 2 [1950] 1956-1997.

ZORZI, Hesiod in the Byzantine and early Renaissance Periods : N. ZoRZI, Hesiod in the Byzantine and early Renaissance Periods, dans : A.C. LONEY / S. SCUlly (éds.), The Oxford Handbook of Hesiod. Oxford 2018, 413-430. 
